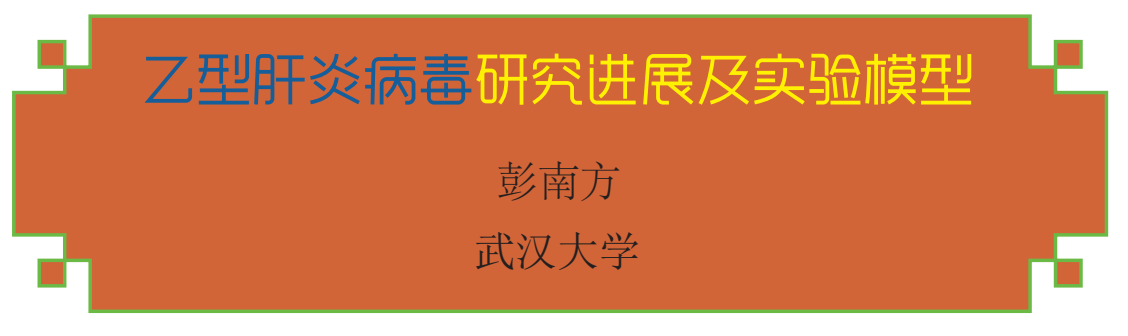

乙型肝炎广泛流行于世界各 国, 是严重威胁人类健康的世界 性疾病之一, 也是我国当前流行 最为广泛、危害性最严重的慢性 疾病，主要侵害儿童以及青壮年。 乙型肝炎病毒 (HBV) 主要通过 血液或者体液特异性地感染人体 肝脏, 在感染过程中若没有被免 疫系统及时清除, 将诱发乙型肝 炎。

乙型肝炎病毒是一种小 DNA
病毒, 其基因组为不完全的双链 DNA 结构。当乙型肝炎病毒感染 宿主细胞后, 其基因组在细胞核 内转变为共价闭合环状 DNA (cccDNA）这个微型染色体作为转录 模板, 负责病毒基因组的后续转 录过程。

乙型肝炎病毒基因组转录 后，会利用宿主的翻译系统表达 一系列的病毒蛋白, 如核心蛋白 ( $\mathrm{HBcAg}$ 或者 $\mathrm{HBeAg})$, 逆转录
酶 (Pol), 包膜蛋白 (HBsAg) 以 及病毒蛋白 $\mathrm{HBx}$ 。目前为止, 世 界上的乙型肝炎病毒研究团队成 功解码了前三种病毒蛋白的主要 功能, 虽然许多研究表明病毒蛋 白 $\mathrm{HBx}$ 在多种细胞活动如细胞 调亡、细胞周期调控以及病毒感 染的过程中发挥功能, 但是对其 最主要的功能研究因缺乏有效的 HBV 感染模型而受限, 也就是病 毒蛋白 HBx 的 “X 档案” 有待进 一步探究补充。

\section{乙型肝炎病毒研究最新 进展}

2016 年 Nature杂志发表了 Decorsière 等研究团队对 HBx 功

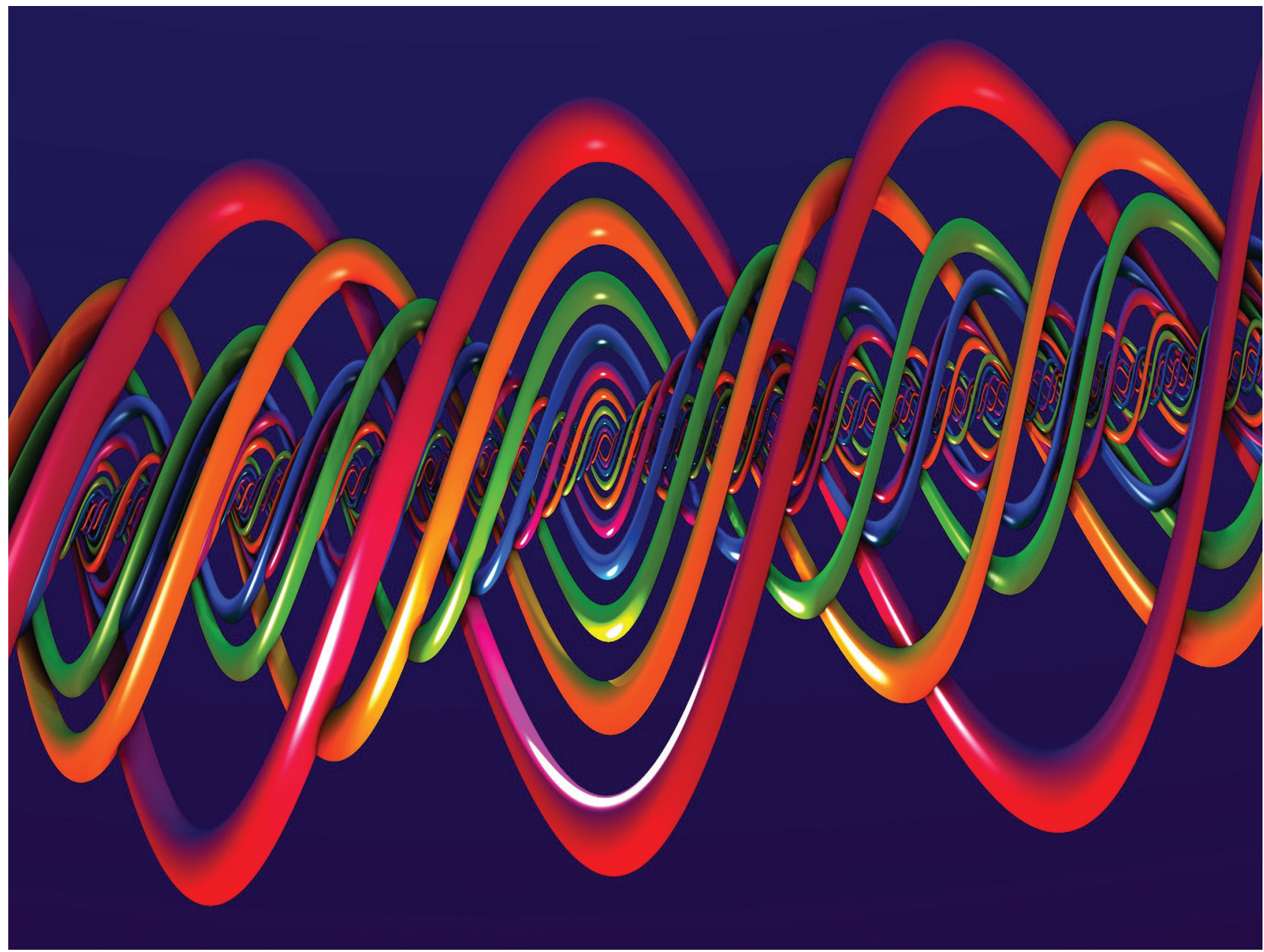


能的最新研究, 首次揭示了病毒 蛋白 HBx 在帮助乙型肝炎病毒逃 逸机体免疫保护机制的过程中发 挥重要作用。

近几年来, 细胞中泛素化 蛋白酶系统在许多重要的细胞活 动中都发挥至关重要的作用, 在 生物学以及医学研究中得到了广 泛关注。我们以 CRL4-DDB1 蛋 白质复合物为例, 简单介绍这个 泛素化 - 蛋白酶系统的工作机制: 蛋白质 CRL4 作为一个 E3 连接 酶, 主要负责用泛素分子标记细 胞内的一些蛋白, 而蛋白质 DDB1 主要负责降解被蛋白质 CRL4 标 记了泛素分子的蛋白质, 从而使 被标记的蛋白质失去正常的生理 功能。

Decorsière团队的研究工作 指出, 乙型肝炎病毒的病毒蛋白 HBx 能够同 CRL4-DDB1 蛋白质 复合物发生相互作用, 特异性地 将泛素酶 CRL4 引导至 Smc5/6 蛋白对其添加泛素分子标记, 最 终诱导 DDB1 蛋白质对 Smc5/6 蛋 白的降解, 从而使其失去正常的 生理功能。在这里我们要指出的 是, $\mathrm{Smc5} / 6$ 蛋白结合在乙型肝炎 病毒的基因组上，抑制乙型肝炎 病毒的转录, 从而保护宿主免受 大量乙型肝炎病毒的侵害。

虽然宿主在病毒感染的过 程中有一系列防御措施, 但是 Decorsière 的研究工作表明, 病 毒也相应地在感染过程中发展了 逃逸宿主监控的措施, 如利用 病毒蛋白 HBx 作为 “间谍”, 将 细胞内的泛素化 - 蛋白酶系统 CRL4-DDB1 蛋白质复合物 “诱 骗” 至 “自己人”Smc5/6 蛋白上, DDB1 蛋白将其 “错杀”, 没有了 Smc5/6 蛋白阻止乙型肝炎病毒基
因组转录, 乙型肝炎病毒大量转 录翻译, 从而对宿主造成进一步 的严重伤害。

Decorsière 等研究结果不仅 提出了乙型肝炎病毒蛋白 HBx 的 新功能, 还暗示了在未来的针对 抗乙型肝炎病毒的新药开发中, 病毒蛋白 $\mathrm{HBx}$ 可以作为一个新的 药物作用靶点。

\section{乙型肝炎病毒研究模型}

发展

\section{细胞模型}

自发现肝炎病毒依赖性, 医 学家和科学家利用多种科学实验 手段对其进行深入研究, 在研究 过程中, 体外实验中涉及到的细 胞模型也经历了一系列发展。

出现最早、目前应用较多的 肝炎病毒研究细胞模型是一系列 从肝癌患者的肿瘤样本中分离出 来的癌细胞系, 如 Huh7, HepG2 等。这类细胞系的优点是能够无 限增殖, 获得比较容易; 但是其 不太支持体外肝炎病毒的感染, 故不能作为一个很好的病毒感染 模型。因此，找到易得且支持肝 炎病毒体外感染的细胞模型是肝 炎病毒研究需要突破的瓶颈。

目前为正，原代人肝细胞被 认为是实验室研究肝炎病毒感染 的最优模型。其获取方式有两种： （一）在肝脏肿瘤患者切除瘤块 时, 肿瘤边缘极少部分正常肝脏 组织可以用来分离原代人肝细胞; （二）在流产的胚胎中, 获取其胎 肝以分离原代人肝脏细胞。原代 人肝细胞在培养过程中存活时间 约 1-2 周, 但是许多科研团队研
究数据表明, 其能够支持乙型肝 炎病毒和丙型肝炎病毒的感染, 是非常成功的细胞感染模型。

考虑到原代人肝细胞获取不 容易, 此外, 由于不同的肝组织 贡献者分离出来的人原代肝细胞 也因贡献者性别、年龄、用药情 况不同, 以及肝脏疾病严重程度 等存在较大差异, 具有分化功能 的干细胞吸引了科学家的视线。

诱导的多功能干细胞是新 开发的、用于生成能成功感染 肝炎病毒的肝细胞的新来源。诱 导的多功能干细胞一旦获得, 可 以稳定地分化成成熟的肝细胞。 Schwartz 等研究团队通过科学实 验发现, 人多功能干细胞分化而 来的肝细胞能够成功为丙型肝炎 病人体内分离的丙型肝炎病毒感 染; 同样, Shlomai 等研究发现 由干细胞诱导分化而来的成熟的 肝细胞也能成功感染 HBV。

当然, 多功能干细胞来源的 肝细胞有自身缺点, 例如, 其不能 分化为功能完全的、成熟的肝细 胞, 但是, 科研人员利用多种方 法解决了这个难题, 例如, 在体外 诱导多功能干细胞分化的过程中 通过添加小分子改变细胞生长微 环境来实现肝细胞功能诱导完全。

此外, 科学家预测, 由于多 功能干细胞体外分化的特性, 可 以在体外诱导干细胞发育成为血 管以及功能齐全的人体肝脏模型, 从而在 $3 \mathrm{D}$ 水平研究具有功能血 管的病毒性肝炎, 从而为肝炎的 研究以及临床治疗提供新的视野。

\section{实验小鼠模型}

在研究肝炎病毒感染的过程 
中, 细胞模型较为单一, 不能反 映肝炎病毒感染后个体水平的病 理进程数据。因此, 找到肝炎病 毒研究中有效的动物模型是病毒 学家和医学家的重要任务。

自 1960s 以来, 科学家发现 黑猩猩个体能够成功感染肝炎病 毒, 很长一段时间以来将其作为 肝炎病毒的研究模型。但是由于 黑猩猩的供应有限, 价格昂贵, 人类与黑猩猩肝病发展进程存在 差异, 以及伦理道德的限制, 其 并非是一个最好的研究模型。

小鼠模型最开始在乙型肝炎 病毒研究的过程中建立: 通过高 压尾静脉注射乙型肝炎病毒的基 因组表达质粒至小鼠中, 检测个 体水平上乙型肝炎病毒感染宿主 免疫反应等方面的关系。由于鼠 源和人源的肝细胞中表达的宿主 因子差异显著, 小鼠肝细胞并不 能真实反应肝炎病毒感染引起的 人体反应, 但值得兴奋的是, 科 学家通过构建人源化的小鼠模 型, 克服了人与鼠物种上的隔阂。

人源化小鼠构建的过程可以 简单概括为: 通过异种移植原代 人肝细胞至免疫缺陷的小鼠体内, 原代人肝细胞紧接着再定位于小 鼠肝脏。为了诱导移植的人肝细 胞取代正常的小鼠肝细胞, 科学 家还要引入一种转基因来控制肝 脏损伤来杀伤鼠自身的肝脏细胞, 从而为移植的人肝脏细胞生长创 造有利的生长条件。

第一种成功构建的人源小鼠 是异种移植了原代人肝细胞的重 症综合性免疫缺陷小鼠, 小鼠的 基因组包含了尿激酶纤维蛋白溶 酶原激活剂转基因, 当这个转基 因被人为激活时, 会造成小鼠急 性肝中毒, 从而使小鼠自身肝细

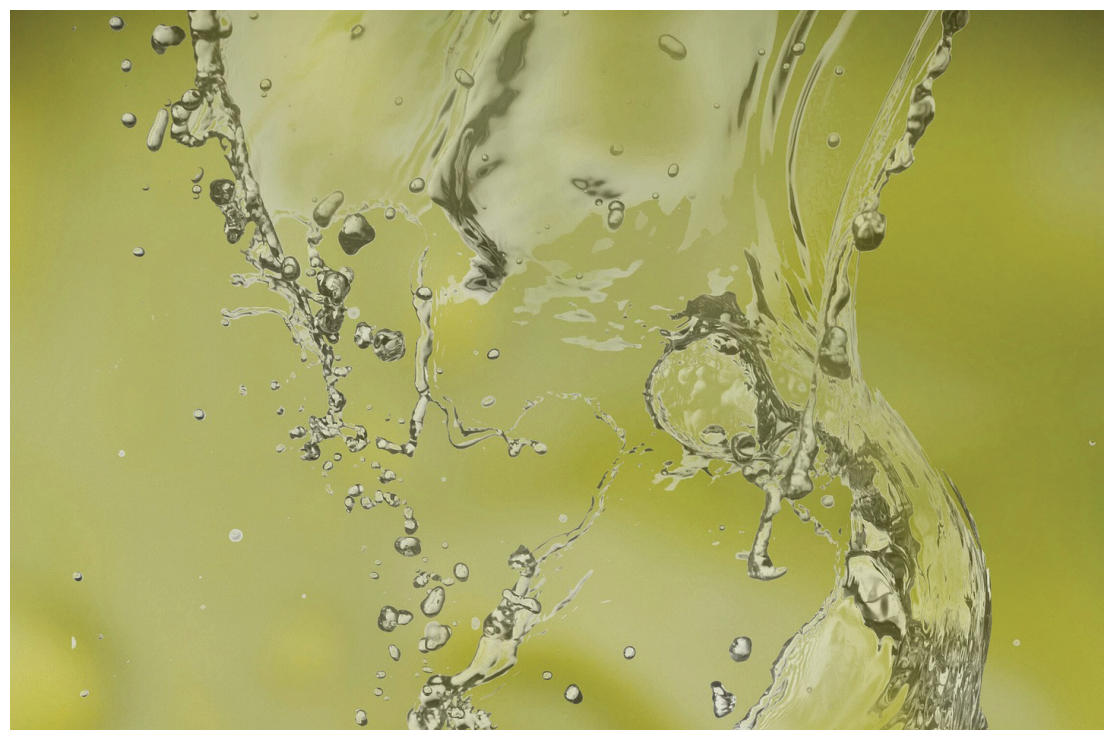

胞坏死, 而人肝细胞获得生存, 这个肝脏人源化的小鼠模型可以 用于科学研究中肝炎病毒的研究。

目前为止, 科学家可以利用 不同的手段诱导鼠自身肝细胞死 亡, 而保留异种移植的原代人肝 细胞生长, 最终获得人源小鼠模 型。例如, Fah 基因敲除小鼠在 一直服用名为尼替西农药物的时 候, 能维护肝脏健康; 一旦停药, 小鼠肝脏细胞特异性产生有毒代 谢物而病变死亡, 而异种移植原 代人肝细胞在此时可以顺利生长 而使小鼠肝脏人源化, 是一个很 好的肝炎病毒研究模型。

此外, 一种名为 AFC8 的小 鼠模型, 其基因组包含了半胱天 冬酶 8 转基因, 在药物 FK506 处 理后, 小鼠肝细胞调亡, 而异种 移植原代人肝细胞可以正常生长。 Washburn 等研究发现, 在肝脏 人源化的 $\mathrm{AFC} 8$ 小鼠模型中, 丙 型肝炎病毒感染能够成功引起小 鼠肝脏的纤维化病变。

人源化小鼠的成功构建将极 大推进科学家和医学家对肝炎病 毒的研究。随着这个模型的完善, 其也可以用于肝炎病毒疫苗的笁
选研究, 为人类病毒性肝炎疾病 的预防和治疗产生深远影响。

参考文献

[1] Decorsière A, Mueller H, van Breugel $\mathrm{P} \mathrm{C}$, et al. Hepatitis $\mathrm{B}$ virus $\mathrm{X}$ protein identifies the Smc5/6 complex as a host restriction factor. Nature, 2016;531(7594):386.

[2] Liang TJ. Virology. The X-Files of hepatitis B. Nature, 2016;531(7594): 313.

[3] Shlomai A, Schwartz RE, Ramanan $\mathrm{V}$, et al. Modeling host interactions with hepatitis B virus using primary and induced pluripotent stem cellderived hepatocellular systems. Proceedings of the National Academy of Sciences of the United States of America, 2014;111(33):12193.

[4] Schwartz RE, Trehan K, Andrus L, et al. Modeling hepatitis $\mathrm{C}$ virus infection using human induced pluripotent stem cells. Proc Natl Acad Sci U S A. 2012;109(7):2544-2548.

[5] Washburn ML, Bility MT, Zhang L, et al. A humanized mouse model to study hepatitis $\mathrm{C}$ virus infection, immune response, and liver disease. Gastroenterology, 2011;140(4):13341344.

[6] Thomas E, Liang TJ. Experimental models of hepatitis B and C - new insights and progress. Nature Reviews Gastroenterology \& Hepatology, 2016;13(6):362-374. 